\title{
Abdominal Gossypibomas: Computed Tomography Scanner Findings of a Series of 15 Cases
}

\author{
Mazamaesso Tchaou ${ }^{1 *}$, Boyodi Tchangai ${ }^{2}$, Djatougbe Fafa Dosseh ${ }^{1}$, Pihou Gbande ${ }^{1}$, \\ Bereza Kolou1, Lantam Sonhaye1, Lama-Kegdigoma Agoda-Koussema1, Victor Adjenou1 \\ ${ }^{1}$ Department of Radiology, University of Lomé, Lomé, Togo \\ ${ }^{2}$ Department of Visceral Surgery, University of Lomé, Lomé, Togo \\ Email: ‘joseph_tchaou@yahoo.fr, botchangai@yahoo.fr, cinafa2@gmail.com,gbandepihou@yahoo.fr, berkolou@yahoo.fr, \\ sonhayelantam@gmail.com, agoda_kousssema@yahoo.fr, kadjenou@yahoo.fr
}

How to cite this paper: Tchaou, M., Tchangai, B., Dosseh, D.F., Gbande, P., Kolou, B., Sonhaye, L., Agoda-Koussema, L.-K. and Adjenou, V. (2020) Abdominal Gossypibomas: Computed Tomography Scanner Findings of a Series of 15 Cases. Open Journal of Radiology, 10, 16-22.

https://doi.org/10.4236/ojrad.2020.101003

Received: January 17, 2020

Accepted: February 24, 2020

Published: February 27, 2020

Copyright ( 2020 by author(s) and Scientific Research Publishing Inc. This work is licensed under the Creative Commons Attribution International License (CC BY 4.0).

http://creativecommons.org/licenses/by/4.0/

\begin{abstract}
Background: "Gossypiboma" or "textiloma" refers to accidental retention of textile material in an operated area of the body. Abdominal surgery is most often responsible for this complication. The purpose of our study was to describe the appearance of abdominal gossypibomas on computed tomography (CT) scan images. Materials and Methods: We conducted a retrospective study over ten years (from January 1, 2009 to December 31, 2018) at the Teaching University Hospital of Lomé. We collected all surgically confirmed cases of abdominal gossypibomas with abdominal CT scans. Results: Fifteen cases were compiled, with a sex-ratio of 0.36 . The average age of the patients was 34 years. The initial surgery was emergent in nine of 15 cases, 11 of which consisted of gynecological interventions. The gossypiboma symptom incubation period was between 3 days and 3 years. A radiopaque marker was observed via CT scans in three of 15 cases. The gossypiboma appeared encapsulated and spongiform in six cases and cystic in seven cases. There were two cases where the gossypiboma migrated into hollow organs. Conclusion: Abdominal gossypibomas appear polymorphic on CT scans and should be considered in patients who present with an abdominal mass and a history of abdominal surgery, even for absent radiopaque markers.
\end{abstract}

\section{Keywords}

Gossypiboma, Textiloma, Computed Tomography, Safety in Surgery, Togo

\section{Introduction}

The term gossypiboma derives from the combination of two words: the Latin 
"gossypium," which means cotton and the Swahili "boma", which means place of concealment [1]. Gossypiboma refers to accidental retention of textile material in the operated area of the body, also called textiloma. Abdominal surgery is often responsible for these complications [2]. Gossypibomas are sources of forensic medical problems due to their associated morbidity and mortality. This morbidity can be compounded by difficulties in diagnosis, specifically with regard to symptom latency and the clinical polymorphisms. These risks justify the use of imaging, often with simple radiographs; however, these are often insufficient [3]. In other cases, computed tomography (CT) scans are useful for refining the diagnosis. We examined the incidence and characteristics of abdominal gossypiboma on CT scans.

\section{Materials and Methods}

This retrospective study was conducted over nearly ten years, from January 1, 2009 to December 31, 2018) at Sylvanus Olympio Teaching Hospital of Lomé (Togo). We included all surgically confirmed cases of abdominal gossypiboma with abdominal CT images before gossypiboma ablation. The variables studied were carried from the medical records and CT-scan archives.

The study was approved by the scientific ethics committee of the University of Lomé and by the Data Protection Board of the Hospital.

\section{Results}

We compiled a total of 15 gossypiboma cases, consisting of 11 females and 4 males, accounting for a sex-ratio of 0.36 ; the overall incidence was one out of every 3550 surgical procedures. On average, the patients were 34 years old (range: 16 - 78 years). The initial surgery was emergent in nine of 15 cases, 11 of which consisted of gynecological interventions. Surgical approaches included transverse suprapubic laparotomy $(\mathrm{n}=11)$, median laparotomy $(\mathrm{n}=3)$, and inguinal incision $(n=1)$. The symptom incubation periods ranged from 3 days to 3 years. Before abdominopelvic CT, six patients underwent unprepared abdominal x-rays, and four underwent abdominal ultrasound, all of which were considered non-contributory. An abdominopelvic CT scan was performed, without any further imaging, in five patients.

During the CT scans, if a radiopaque marker was present in the compress, diagnosis was made easier when there were linear foreign bodies, usually of high-density, and round or spiral in shape. In three out of 15 cases, hardened artifacts were observed, in the form of a flash around the material (Figure 1). None of these three patients underwent further imaging, other than the CT scan.

Other forms of gossypiboma, not related to the presence or absence of a radiopaque marker, were observed and can be sub-divided into three groups. The first form included masses with a peripheral shell, regular external limits, and no densification of the surrounding fat. The interiors of these masses consisted of dense meshes, with air and calcified material appearing as an encapsulated 
sponge and described as "spongiform." This form corresponded to a true granuloma, somewhat old, on a gossypiboma, and was observed in six of 15 cases. It was observed in all patients whose initial surgeries were performed more than 3 months prior to imaging (Figure 2).

The second form was observed in two patients who underwent surgeries more than 3 months before. Here, the gossypiboma appeared cystic, and manifested as a hypodense mass (fluid density) with a thick wall and well-defined external limits, forming a real pseudocyst around the gossypiboma (Figure 3).

The third form consisted of mixed lesions, all observed within a short period of 3 days to 3 months. Occasionally, these masses appeared spongy with poorly limited contours, fluid-filled and organized in contact with the textile and forming a real abscess, or with increased density of nearby peritoneal fat and-sometimes-free peritoneal effusion (forming peritonitis). These forms were observed in seven cases.

Besides these classifiable forms, there were two cases of gossypiboma migration into a hollow organ. The first one involved migration of a compress into the transverse colon, simulating a tumor in the scanner. The second case was a pelvic abscess with a fistula wherein the compress migrated into the bladder (Figure 4).

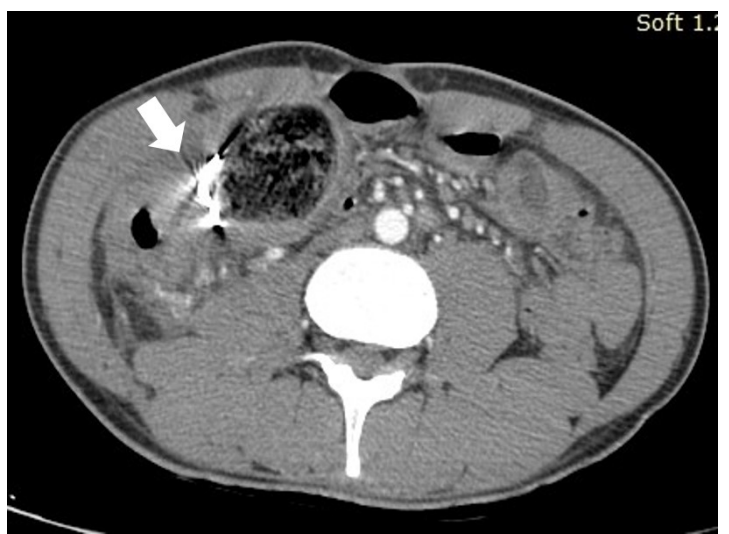

Figure 1. Gossypiboma with a radiopaque marker. Abdominal transversal CT-scan image shows a foreign body (arrow) of very high density in a mass.

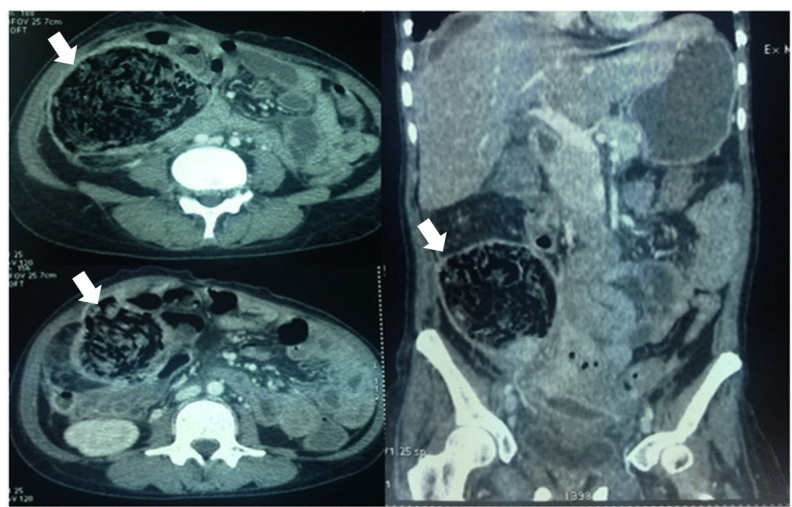

Figure 2. "Spongiform.” Gossypiboma. 


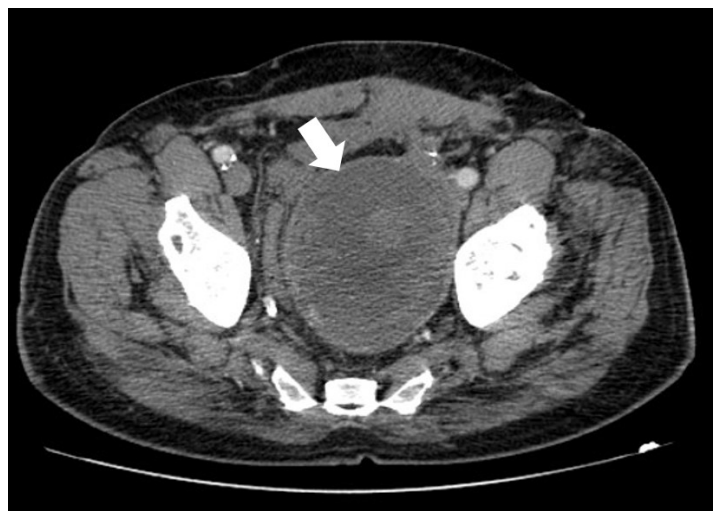

Figure 3. Pseudo cyst-like gossypiboma.

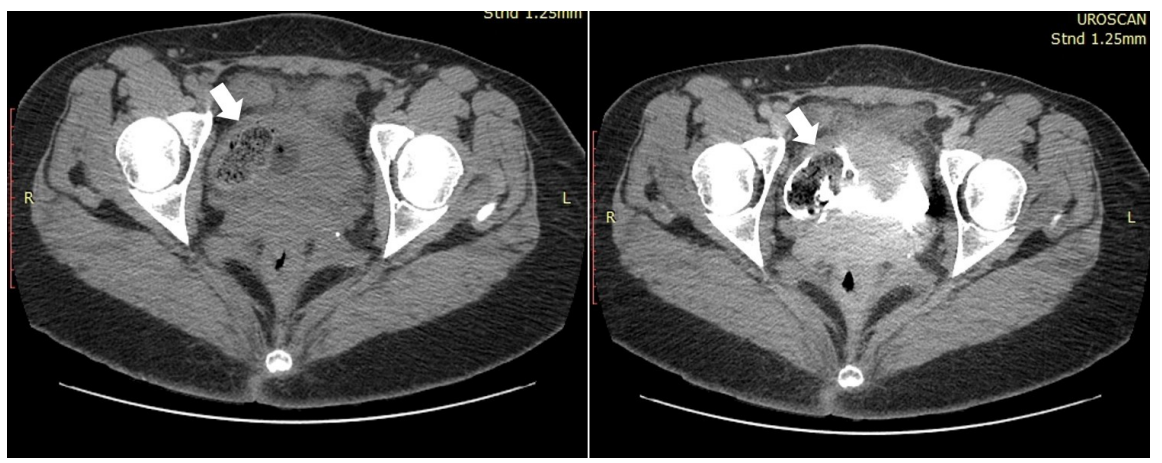

Figure 4. Gossypiboma migration in bladder.

\section{Discussion}

Gossypibomas are the most frequently forgotten foreign bodies after surgery [4] [5] [6] [7] [8]. During abdominal surgeries, their frequency is estimated between 1 in 1000 and 1 in 5000 [8]. At the Teaching University Hospital of Lomé, gossypibomas were noted in one out of every 3550 abdominopelvic surgeries.

Most $(\mathrm{n}=11)$ cases were female, in agreement with previous reports [9] [10] [11]. This female predominance could be explained by the type of incision performed during the gynecological interventions that constituted the main initial surgeries. The suprapubic incision, firstly transversal, is frequently used during these procedures and does not expose the entire abdominal cavity, thereby increasing the risk of gossypiboma. Emergent surgeries appear to be the most common cause of gossypibomas [7] [8], as was the case in six out of 15 cases in this study.

Imaging technique is typically selected based on clinical signs. During the immediate post-surgical period, a simple unprepared abdominal x-ray is adequate when the compresses used during surgery are marked. This was the most commonly used means of detecting gossypibomas [12]. Among our cases, 12/15 compresses did not have radiopaque markings, which is a major flaw in the safety of surgical procedures. The use of labeled compresses, like the textile count, is one measure which may prevent gossypibomas and their sequelae [13] [14]. The clinical expression of gossypibomas can be delayed in onset and diffi- 
cult to diagnose. As a result, clinicians do not always consider gossypiboma as a potential diagnosis and may therefore request non-radiographic examinations. Ultrasound, though quick and non-invasive, may fail to detect gossypiboma amidst abdominal gases [15].

The CT scan is the most useful test for diagnosing gossypiboma and its complications [10] [16]. In practice, CT scans are usually ordered to rule out conditions other than gossypibomas. Other times, CT scans are secondary examinations that are ordered after other techniques fail to reveal a diagnosis. CT scans assist diagnosis when there are no radiopaque markings, and when ultrasound has failed.

Different features are evaluated on CT. A main feature relates to the marking of the textile. These typically consist of high-density linear images where the gossypiboma is immediately apparent. In these cases, the gossypiboma can be observed on an abdomen radiograph that is performed without preparation, or even by using the scout-view on radiography.

The other forms can be sub-divided into three types, which we have correlated with the type of foreign body reaction.

On the anatomopathological level, two forms of gossypibomas have been described. These forms correspond to two types of bodily reactions to the presence of a gossypiboma. Such reactions include either an exudative inflammatory reaction with an abscess or an aseptic and fibrotic cotton reaction with mass development [17].

Anatomopathological aspects of these forms appear distinct on CT scans. Granulomas correspond to spongy and pseudocystic forms, a late revelation which can even simulate an abdominal tumor [9] [10] [15] [16]. Forms with exudative inflammatory reactions correspond to the mixed forms in the scanner, expressing abscesses and peritonitis, and usually appearing soon after the first surgery.

CT can also be used to diagnose complications of gossypiboma, the most common of which involves migration into a hollow organ. This occurs most often in the digestive tract, but also in the bladder-as with one of our patientsor the genital tract [1] [18] [19] [20] [21] [22]. In the majority of cases, gossypiboma is a surgical surprise; the pre-operative diagnosis is for a fecaloma or tumor [15] [21] [23]. The diagnosis can be rectified if the history of surgery is communicated to the radiologist.

\section{Conclusion}

Abdominal gossypibomas appear polymorphic on CT scans and should be considered in any patient with an abdominal mass and a history of abdominal surgery, even for absent radiopaque markers. Prevention remains the best way to avoid gossypibomas and their related morbidity.

\section{Data Availability}

The datasets used and analyzed during the current study are available from the 
corresponding author on reasonable request.

\section{Conflicts of Interest}

The authors have no conflicts of interest to declare.

\section{Funding}

This research did not receive any specific grant from funding agencies in the public, commercial, or not-for-profit sectors.

\section{References}

[1] Patil, K.K., Patil, S.K., Gorad, K.P., Panchal, A.S., Arora, S.S. and Gautam, R.P. (2010) Intraluminal Migration of Surgical Sponge: Gossypiboma. Saudi Journal of Gastroenterology, 16, 221-222. https://doi.org/10.4103/1319-3767.65195

[2] Fernandez Lobato, R., Marin Lucas, F.J. and Fradejas Lopez, J.M. (1998) Postoperative Textilomas: Review of 14 Cases. International Surgery, 83, 63-66.

[3] Choi, B.I., Kim, S.H., Yu, E.S., et al. (1988) Retained Surgical Sponge: Diagnosis with CT and Sonography. American Journal of Roentgenology, 150, 1047-1050. https://doi.org/10.2214/ajr.150.5.1047

[4] Stawicki, S.P., Evans, D.C., Cipolla, J., et al. (2009) Retained Surgical Foreign Bodies: A Comprehensive Review of Risks and Preventive Strategies. Saudi Journal of Gastroenterology, 98, 8-17. https://doi.org/10.1177/145749690909800103

[5] Birolini, D.V., Rasslan, S. and Utiyama, E.M. (2016) Unintentionally Retained Foreign Bodies after Surgical Procedures. Analysis of 4547 Cases. Revista do Colégio Brasileiro de Cirurgióes, 43, 12-17. https://doi.org/10.1590/0100-69912016001004

[6] Yildirim, S., Tarim, A., Nursal, T.Z., et al. (2006) Retained Surgical Sponge (Gossypiboma) after Intra-Abdominal or Retroperitoneal Surgery: 14 Cases Treated at a Single Center. Langenbeck's Archives of Surgery, 391, 390-395. https://doi.org/10.1007/s00423-005-0581-4

[7] Sakorafas, G.H., Sampanis, D., Lappas, C., Papantoni, E. and Christodoulou, S. (2010) Retained Surgical Sponges: What the Practicing Clinician Should Know. Langenbeck's Archives of Surgery, 395, 1001-1007. https://doi.org/10.1007/s00423-010-0684-4

[8] Gawande, A.A., Studdert, D.M., Orav, E.J., Brennan, T.A. and Zinner, M.J. (2003) Risk Factors for Retained Instruments and Sponges after Surgery. The New England Journal of Medicine, 348, 229-235. https://doi.org/10.1056/NEJMsa021721

[9] Coche, G., Pardonnet, M., Chanois, A.M., Rohmer, P., Weill, F.S., Etienne, G. and Didier, D. (1988) Ultrasonography and X-Ray Computer Tomography in the Diagnosis of Intra-Abdominal Textiloma. A Propos of 12 Cases. Journal of Radiology, 69, 243-251.

[10] Tchangai, B., Tchaou, M., Kassegne, I. and Simlawo, K. (2017) Incidence, Root Cause, and Outcomes of Unintentionally Retained Intraabdominal Surgical Sponges: A Series from Two Hospitals in Togo. Patient Safety in Surgery, 11, 25. https://doi.org/10.1186/s13037-017-0140-2

[11] Mathew, R.P., Thomas, B., Basti, R.S. and Suresh, H.B. (2016) Gossypibomas, a Surgeon's Nightmare-Patient Demographics, Risk Factors, Imaging and How We Can Prevent It. The British Journal of Radiology, 90, Article ID: 20160761. https://doi.org/10.1259/bjr.20160761 
[12] Kumar, G.V.S., Ramani, S., Mahajan, A., Jain, N., Sequeira, R. and Thakur, M. (2017) Imaging of Retained Surgical Items: A Pictorial Review Including New Innovations. The Indian Journal of Radiology and Imaging, 27, 354-361.

[13] Stahel, P.F., Mehler, P.S., Clarke, T.J. and Varnell, J. (2009) The 5th Anniversary of the "Universal Protocol": Pitfalls and Pearls Revisited. Patient Safety in Surgery, 3, 14. https://doi.org/10.1186/1754-9493-3-14

[14] Panesar, S.S., Cleary, K., Sheikh, A. and Donaldson, L. (2009) The WHO Checklist: A Global Tool to Prevent Errors in Surgery. Patient Safety in Surgery, 3, 9. https://doi.org/10.1186/1754-9493-3-9

[15] Doh, K., Thiam, I., Takin, R.C.A., Bissirou, I. and Woto, G. (2017) Un cas de textilome renal simulant une tumeur de découverte anatomopathologique. African Journal of Urology, 23, 364-367. https://doi.org/10.1016/j.afju.2016.12.002

[16] Sozutek, A., Colak, T., Reyhan, E., Turkmenoglu, O. and Akpinar, E. (2015) Intra-Abdominal Gossypiboma Revisited: Various Clinical Presentations and Treatments of this Potential Complication. Indian Journal of Surgery, 77, 1295-300. https://doi.org/10.1007/s12262-015-1280-1

[17] Olnick, H.M., Weens, H.S. and Robers, J.V. (1955) Radiological Diagnosis of Retained Surgical Sponges. Journal of the American Medical Association, 159, 1525-1527. https://doi.org/10.1001/jama.1955.02960330025008

[18] Dux, M., Ganten, M. and Lubienski, A. (2002) Retained Surgical Sponge with Migration into the Duodenum and Persistent Duodenal Fistula. European Radiology, 12, S74-S77. https://doi.org/10.1007/s00330-002-1408-0

[19] Agrawal, H., Gupta, N., Krishengowda, U., Gupta, A.K., Naskar, D. and Durga, C.K. (2018) Transmural Migration of Gossypiboma: A Rare Cause of Acute Abdomen. Indian Journal of Surgery, 80, 84-86. https://doi.org/10.1007/s12262-017-1660-9

[20] Butt, U.I., Shafiq, A.B., Umar, M., Ashfaq, M. and Ayyaz, M. (2018) Transmigration and Spontaneous Passage of a Gossypiboma Documented on Contrast Study. Annals of Medicine and Surgery, 38, 42-44. https://doi.org/10.1016/j.amsu.2018.10.022

[21] Kassi, A.B.F., Yenon, K.S. and Koffi, E.M. (2018) A Transmural Migration of a Gossypiboma in the Right Colon Responsible for a Mass Which Mimicked an Abscessed Colonic Tumor: A Case Report. International Journal of Surgery Case Reports, 51, 228-230. https://doi.org/10.1016/j.ijscr.2018.08.046

[22] Javanmard, B., Yousefi, M.R., Fadavi, B. and Fallah Karkan, M. (2017) Retained Surgical Gauze Presenting With Gross Hematuria: A Case Report. Urology Journal, 14, 5027-5029.

[23] Eken, H., Soyturk, M., Balci, G., et al. (2016) Gossypiboma Mimicking a Mesenchymal Tumor: A Report of a Rare Case. American Journal of Case Reports, 17, 27-30. https://doi.org/10.12659/AJCR.896717 